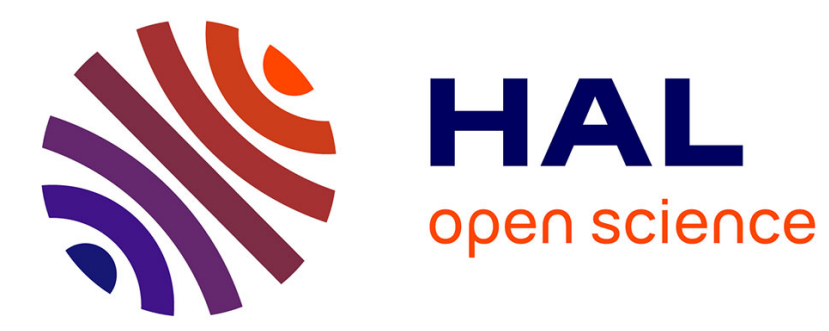

\title{
On Hyper Exponential Stabilization of Linear State-Delay Systems
}

\author{
Andrey Polyakov, Denis Efimov, Emilia Fridman, Wilfrid Perruquetti, \\ Jean-Pierre Richard
}

\section{- To cite this version:}

Andrey Polyakov, Denis Efimov, Emilia Fridman, Wilfrid Perruquetti, Jean-Pierre Richard. On Hyper Exponential Stabilization of Linear State-Delay Systems. Conference on Decision and Control 2017, Dec 2017, Melbourne, Australia. hal-01587780

\section{HAL Id: hal-01587780 \\ https://hal.inria.fr/hal-01587780}

Submitted on 14 Sep 2017

HAL is a multi-disciplinary open access archive for the deposit and dissemination of scientific research documents, whether they are published or not. The documents may come from teaching and research institutions in France or abroad, or from public or private research centers.
L'archive ouverte pluridisciplinaire HAL, est destinée au dépôt et à la diffusion de documents scientifiques de niveau recherche, publiés ou non, émanant des établissements d'enseignement et de recherche français ou étrangers, des laboratoires publics ou privés. 


\title{
On Hyper Exponential Stabilization of Linear State-Delay Systems
}

\author{
Andrey Polyakov, Denis Efimov, Emilia Fridman, Wilfrid Perruquetti and Jean-Pierre Richard
}

\begin{abstract}
A control design algorithm for hyper exponential stabilization of multi-input multi-output linear control system with state-delays is presented based on method of Implicit Lyapunov-Krasovskii Functional (ILKF). The procedure of control parameters tuning is formalized by means of Linear Matrix Inequalities (LMIs). The theoretical results are supported with numerical simulations.
\end{abstract}

\section{INTRODUCTION}

Convergence rate is one of control performance indexes, which quantitatively characterizes the transient speed of a controlled process. Its tuning is always required in order to fulfill some time constraints of control system. From mathematical point of view convergence rate is well specified in the context of the stability theory [1]. Indeed, exponential stability determine the convergence rate of linear ordinary differential equations. To define "fast" control this paper uses linear system as the reference point for comparison of convergence rate. Namely, a nonlinear system is said to be fast if it demonstrates transients motions faster than any linear one, i.e. if the convergence rate of the nonlinear system is faster than any exponential. In [2] such systems were called hyper exponential. The so-called finite-time and fixed-time stable nonlinear systems (see, e.g. [3], [4], [5], [6], [7], [8], [9], [10]) are also locally/globally hyper exponentially stable.

Modern control theory basically deals with control of mathematical models, which are just an approximation of a real (physical) plant under some assumptions on its behavior. Linear models are still the most popular way to describe behavior of a plant. Time delays are usual effects in control systems [11], [12], [13], [14]. They appear in the form of transport delays (due to a finite speed of propagation of information) as well as models of actuators or due to approximation of models of mathematical physics described by partial differential equations.

This paper presents a control design algorithm for hyper exponential stabilization of a multi-input multi-output linear control system with state-delays. The ILKF method is utilized for this purpose. To design a control law, which realizes global hyper exponential stabilization of the statedelay system we use two properly adapted ILKF. This paper

The paper is partially supported by ANR Finite4SoS and Russian Science Foundation under grant 17-19-01422 (in Section V).

Andrey Polyakov, Denis Efimov, Wilfrid Perruquetti and Jean-Pierre Richard are with Inria Lille, 40. av Halley, Villenueve d'Ascq, France, (email: andrey.polyakov(denis.efimov, wilfrid.perruquetti)@inria.fr).

Emilia Fridman is with Department of Electrical Engineering and Systems, Tel Aviv University, 69978, Israel (e-mail: emilia@eng.tau.ac.il)

Andrey Polyakov and Denis Efimov are also with ITMO University, 49 Kronverkskiy av., 197101, Saint-Petersburg, Russia

Wilfrid Perruquetti and Jean-Pierre Richard are also with Ecole Centrale de Lille, Villenueve d'Ascq, France(e-mail: jean-pierre.richard@ec-lille.fr). extends the results of [2] and relaxes the restrictions to the plant model. The key differences are as follows: the problem of global hyper exponential stabilization is studied; the control design procedure is presented for multi input system; the restriction to the matrix of the state-delayed term is relaxed; the issues of practical implementation of the obtained implicit control law are also studied. In particular, we present a sampled approach to ILKF-based control realization and we prove that it is robust with respect to variation of sampling period.

Notation: $\mathbb{R}$ is the field of real numbers, $\mathbb{R}_{+}=\{x \in \mathbb{R}$ : $x>0\} ; \mathbb{N}$ is the set of natural numbers; $\|\cdot\|$ is the Euclidian norm in $\mathbb{R}^{n} ; C(X, Y)$ is the space of continuous maps $X \rightarrow$ $Y$, where $X, Y$ are some subsets of finite dimensional spaces; $C_{h}=C\left([-h, 0], \mathbb{R}^{n}\right)$ and $C_{h}^{0}:=\left\{\varphi \in C_{h}: \varphi(0)=0\right\}$ is the linear subspace of $C_{h} ;\|\cdot\|_{h}$ is the uniform norm in $C_{h}$; $I_{n} \in \mathbb{R}^{n \times n}$ - the identity matrix; $\mathbf{0}$ denotes zero element, e.g. $\mathbf{0} \in \mathbb{R}^{n}$ is the zero vector but $\mathbf{0} \in C_{h}$ is the zero-valued function; $\operatorname{diag}\left\{\lambda_{1}, . ., \lambda_{n}\right\}$ - diagonal matrix; positive definite continuous function $\sigma: \mathbb{R} \rightarrow \mathbb{R}$ belongs to the class $\mathcal{K}$ if it is strictly increasing on $\mathbb{R}_{+}$and $\sigma(0)=0$; if additionally it is radially unbounded then $\sigma$ belongs to $\mathcal{K}_{\infty}$.

\section{Problem Statement}

\section{A. System Description and Basic Assumptions}

Let us consider the linear state-delay control system

$$
\begin{gathered}
\dot{x}(t)=A x(t)+L x(t-h)+B u(t), \\
x(\tau)=\varphi_{0}(\tau), \quad \tau \in[-h, 0],
\end{gathered}
$$

where $x(t) \in \mathbb{R}^{n}$ is the vector of the current state, $u(t) \in$ $\mathbb{R}^{m}$ is the control input, $A, L \in \mathbb{R}^{n \times n}$ are system matrices, $B \in \mathbb{R}^{n \times m}$ is the matrix of control gains, $h>0$ is the state delay, $\varphi \in C_{h}$ is the vector-valued function, which defines the initial state of the time-delay system.

Assumption 1: We assume that all parameters of the system (1) are known, the pair $\{A, B\}$ is controllable and the matrix $B$ is of full column rank, i.e. $\operatorname{rank}(B)=m \leq n$.

An additional assumption concerning the matrix $L$ is be given below (see Assumption 2).

\section{B. Control aim}

We restrict a class of admissible feedback control laws to continuous operators $\tilde{u}: C_{h} \rightarrow \mathbb{R}^{m}$ such that

$$
u(t)=\tilde{u}\left(x_{h}(t)\right), \quad \tilde{u}(\mathbf{0})=\mathbf{0},
$$

where $x_{h}(t) \in C_{h}, x_{h}(t)(\theta)=x(t+\theta),-h \leq \theta \leq 0$ is the distributed state of the time delay system (1). 


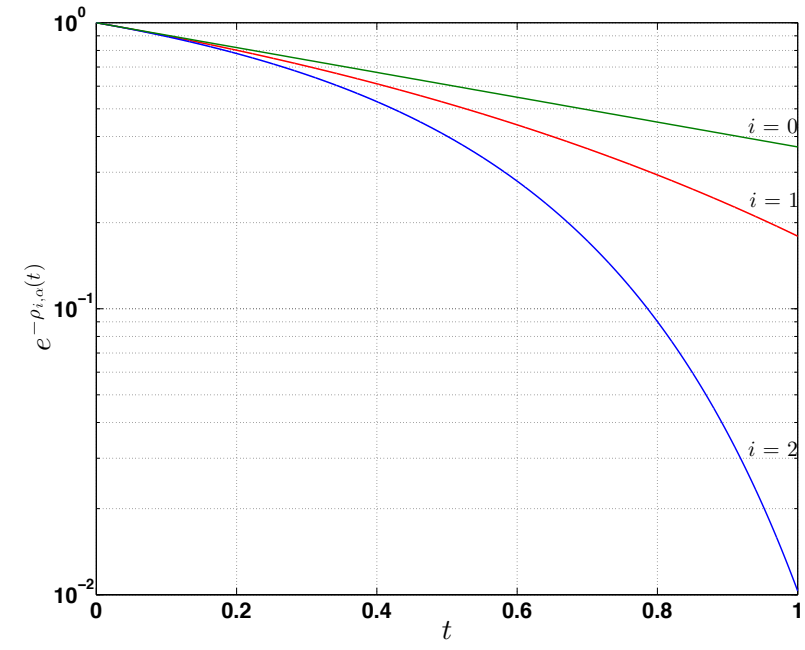

Fig. 1. Hyper exponential rate of convergence

The goal of the paper is to design a control law $\tilde{u}$, which stabilizes the origin of the system with a hyper exponential convergence rate in the sense of Definition 1 .

Given vector $\alpha=\left(\alpha_{0}, \alpha_{1}, \ldots, \alpha_{r}\right)^{\top} \in \mathbb{R}_{+}^{r+1}$ with $r \geq 0$ let us define recursively the following family of functions

$$
\rho_{0, \alpha}(s)=\alpha_{0} s, \quad \rho_{i, \alpha}(s)=\alpha_{i}\left(e^{\rho_{i-1, \alpha}(s)}-1\right),
$$

where $i=1,2, \ldots, r$. Obviously $\rho_{i, \alpha}(0)=0$. The Fig. 1 depicts $e^{-\rho_{i, \alpha}(t)}$ with $t>0$ for $i=0,1,2$ and $\alpha_{i}=1$ in the logarithmic scale in order to show a difference of decay rates. The exponential decay rate corresponds to the straight line $(i=0)$ in the logarithmic scale. The considered functions decrease faster than any exponential one if $i \geq 1$.

Definition 1 (Rated Hyper Exponential Stability, [2]):

The origin of the closed-loop system (1) is said to be globally hyper exponentially stable of degree $r \in \mathbb{N}$ with the convergence rate $\alpha \in \mathbb{R}_{+}^{r+1}$ if there exists $\beta \in \mathcal{K}_{\infty}$ :

$$
\left\|x_{h}(t)\right\|_{h} \leq \beta\left(\left\|\varphi_{0}\right\|_{h}\right) e^{-\rho_{r, \alpha}(t)}, \quad t>0 .
$$

For $r=0$ the latter definition gives the exponential stability.

\section{PRELIMINARIES}

\section{A. Method of Implicit Lyapunov-Krasovskii Functional}

Below we utilize a special class of functions introduced in the following definition. Denote $\Gamma=\left\{(\sigma, s) \in \mathbb{R}_{+}^{2}: q(\sigma, s)=0\right\}$.

Definition 2: [2] The function $q: \mathbb{R}_{+}^{2} \rightarrow \mathbb{R},(\sigma, s) \rightarrow$ $q(\sigma, s)$ is said to be of the class $\mathcal{I K}_{\infty}$ iff

1) $q$ is continuous on $\mathbb{R}_{+}^{2}$;

2) $\forall s \in \mathbb{R}_{+} \exists \sigma \in \mathbb{R}_{+}: q(\sigma, s)=0$;

3) the function $q(\cdot, s)$ is strictly decreasing if $s \in \mathbb{R}_{+}$is fixed;

4) the function $q(\sigma, \cdot)$ is strictly increasing if $\sigma \in \mathbb{R}_{+}$is fixed;

5) $\lim _{0=0,} \sigma \lim _{\sigma \rightarrow 0^{+}} s=0$ and $\lim _{s \rightarrow+\infty} \sigma=+\infty$.

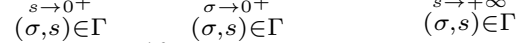

If $q \in \mathcal{I} \mathcal{K}_{\infty}$ then (see, [2]) there exists a unique function $\sigma \in \mathcal{K}_{\infty}$, such that $q(\sigma(s), s)=0$ for all $s \in \mathbb{R}_{+}$.

Recall that the operator $g: \mathbb{Z} \rightarrow \mathbb{Y}$, where $\mathbb{Z}$ and $\mathbb{Y}$ are Banach spaces, is called $F$-differentiable (Frèchet differentiable) at $z_{0} \in \mathbb{Z}$ if there exists a linear bounded operator $D g_{z_{0}}: \mathbb{Z} \rightarrow \mathbb{Y}$ such that

$$
\frac{\left\|g(z)-g\left(z_{0}\right)-D g_{z_{0}}\left(z-z_{0}\right)\right\|_{\mathbb{Y}}}{\left\|z-z_{0}\right\|_{\mathbb{Z}}} \rightarrow 0 \quad \text { as } \quad\left\|z-z_{0}\right\|_{\mathbb{Z}} \rightarrow 0
$$

where $\|\cdot\|_{\mathbb{Z}}$ and $\|\cdot\|_{\mathbb{Y}}$ are norms in the Banach spaces $\mathbb{Z}$ and $\mathbb{Y}$, respectively.

Theorem 1: [2] If there exists a continuous functional $Q$ : $\mathbb{R}_{+} \times C_{h} \rightarrow \mathbb{R}$ such that:

C1) $Q$ is continuously $F$-differentiable on $\mathbb{R}_{+} \times C_{h}$;

$C 2)$ for any $y \in C_{h}$ there exists $V \in \mathbb{R}_{+}$such that $Q(V, y)=0$; C3) $\frac{\partial Q(V, y)}{\partial V}<0$ for all $V \in \mathbb{R}_{+}$and $y \in C_{h}$;

C4) there exist $q_{i} \in \mathcal{I} \mathcal{K}_{\infty}, i=1,2$ such that for all $V \in \mathbb{R}_{+}$

$$
\begin{aligned}
q_{1}(V,\|y(0)\|) \leq Q(V, y), & \forall y \in C_{h} \backslash C_{h}^{0}, \\
Q(V, y) \leq q_{2}\left(V,\|y\|_{h}\right), & \forall y \in C_{h} \backslash\{\mathbf{0}\},
\end{aligned}
$$

$C 5)$ for all $\left(V, x_{h}(t)\right) \in \Omega$ with $x(t)$ satisfying (1) we have

$$
\frac{\partial Q\left(V, x_{h}(t)\right)}{\partial t} \leq-\sigma(\|x(t)\|), \quad \forall t \in \mathbb{R}_{+},
$$

where $\sigma: \mathbb{R} \rightarrow \mathbb{R}$ and

$$
\Omega=\left\{(V, y) \in \mathbb{R}_{+} \times C_{h}: Q(V, y)=0\right\} .
$$

Then the origin of the system (1) is Lyapunov stable if $\sigma$ is a nonnegative function and asymptotically stable if $\sigma \in \mathcal{K}$.

It is worth stressing that $V$ is treated as a scalar parameter in all conditions of Theorem 1 . However, the conditions C1)C4) allow the Implicit Function Theorem [15] to be applicable to the equation $Q(V, \varphi)=0$ that guarantees the existence of a positive definite functional $V: C\left([-h, 0], \mathbb{R}^{n}\right) \rightarrow$ $\mathbb{R}: Q(V(\varphi), \varphi)=0$ satisfying all standard conditions $\sigma_{1}(\|\varphi(0)\|) \leq V(\varphi) \leq \sigma_{2}\left(\|\varphi\|_{h}\right)$ required for a LyapunovKrasovskii functional.

Definition 3: A functional $Q$ satisfying the conditions C1)-C4) is called an ILKF candidate.

Implicit Lyapunov-Krasovskii functional-based analysis of exponential and hyper exponential stability requires some additional restrictions to the class of functionals.

Corollary 1: Let $Q_{1}, Q_{2}$ be ILKF candidates and $\left.C 4^{*}\right) \exists c>0$ such that $q_{11}(c s, s) \geq 0$ if $s<1$ and $q_{12}(c s, s) \geq 0$ if $s>1$, where $q_{1 i}$ is defined by the condition C4) of Theorem 1 for $Q_{i}, i=1,2$;

$\left.C 5^{*}\right)$ for $\left(V, x_{h}(t)\right) \in \Omega$ such that $x(t)$ satisfies (1) one has

$\frac{\partial Q_{i}\left(V, x_{h}(t)\right)}{\partial t} \leq 2 \alpha_{0} V \ln \left(e V^{(-1)^{i}}\right) \frac{\partial Q_{i}\left(V, x_{h}(t)\right)}{\partial V}, \quad \forall t \in \mathbb{R}_{+}$,

where $\alpha_{0}>0$ and $i=1,2$;

$\left.C 6^{*}\right)$ for $y \in C_{h}$ one has $Q_{1}(1, y)=Q_{2}(1, y)$,

then the origin of system (1) is globally hyper exponentially stable with degree $r=1$ and convergence rate $\alpha=\left(\alpha_{0}, 1\right)$.

Proof. Let us consider the functional $V: C_{h} \rightarrow \mathbb{R}_{+}$defined implicitly as a solution to $Q_{i}(V, \varphi)=0, \varphi \in C_{h}$ where $i=1$ if $Q_{1}(1, \varphi)<0$ and $i=2$ otherwise. Due to monotonicity condition C3) (see Theorem 1) and the condition $C 6^{*}$ ) we derive that $\left\{\varphi \in C_{h}: Q_{1}(1, \varphi)<0\right\}=\left\{\varphi \in C_{h}\right.$ : $\left.Q_{2}(1, \varphi)<0\right\}$. From C3) and C*6) one has $Q_{1}(V(\varphi), \varphi)=0$ if $V(\varphi) \leq 1$ and $Q_{2}(V(\varphi), \varphi)=0$ if $V(\varphi) \geq 1$.

Taking into account Condition $C 5^{*}$ ) and the Implicit Function [15] we derive

$$
\begin{gathered}
\dot{V}\left(x_{h}(t)\right)=-\left.\left[\frac{\partial Q_{i}\left(V, x_{h}(t)\right)}{\partial V}\right]^{-1} \frac{\partial Q_{i}\left(V, x_{h}(t)\right)}{\partial V}\right|_{\left(V, x_{h}(t)\right) \in \Omega}= \\
-2 \alpha_{0} V\left(x_{h}(t)\right)\left(1+(-1)^{i} \ln \left(V\left(x_{h}(t)\right)\right),\right. \\
\text { where } i=1 \text { if } V\left(x_{h}(t)\right)<1 \text { and } i=2 \text { if } V\left(x_{h}(t)\right)>1 . \text { The }
\end{gathered}
$$


function $V\left(x_{h}(\cdot)\right): \mathbb{R}_{+} \rightarrow \mathbb{R}_{+}$has no classical derivative at $t_{1} \in \mathbb{R}_{+}: V\left(x_{h}\left(t_{1}\right)\right)=1$, but it has negative upper righthand derivative implying isolation of such a time instant $t_{1}$.

The obtained estimate yields $V\left(x_{h}(t)\right) \leq V\left(\varphi_{0}\right) e^{-\rho_{1, \alpha}(t)}$ with $\alpha=\left(\alpha_{0}, 1\right)$. Finally, the condition $\left.C 4^{*}\right)$ implies $c\|\varphi(0)\| \leq V(\varphi)$, i.e. $\left\|x_{h}(t)\right\| \leq c^{-1} V\left(\varphi_{0}\right) e^{-\rho_{1, \alpha}(t)}$.

\section{B. ILKF Candidates for Linear State-Delay System}

Let us consider the linear dilations (see, [16]) in $\mathbb{R}^{n}$

$$
\mathfrak{d}(\lambda)=e^{G_{\mathfrak{o}} \ln \lambda} \quad \text { with } \quad \lambda>0,
$$

where the matrix $G_{\mathfrak{d}} \in \mathbb{R}^{n \times n}$ is known as the generator of $\mathfrak{d}$ satisfying, obviously, the following identity

$$
\frac{d}{d \lambda} \mathfrak{d}(\lambda)=\frac{1}{\lambda} G_{\mathfrak{d}} \mathfrak{d}(\lambda), \quad \lambda>0 .
$$

Lemma 1: Let $\mathfrak{d}_{i}$ be two linear dilations $(i=1,2)$ of the form (7) and the nonlinear functionals $Q_{i}: \mathbb{R}_{+} \times$ $C\left([-h, 0], \mathbb{R}^{n}\right) \rightarrow \mathbb{R}$ be defined as follows

$$
\begin{gathered}
Q_{i}(V, \varphi)=-1+\varphi(0)^{\top} \mathfrak{d}_{i}\left(\frac{1}{V}\right) P \mathfrak{d}_{i}\left(\frac{1}{V}\right) \varphi(0)+ \\
\int_{-h}^{0}\left(e V^{(-1)^{i}}\right)^{\frac{\mu(2 \tau+h)}{h}} \varphi^{\top}(\tau) \mathfrak{d}_{i}\left(\frac{1}{V}\right) R \mathfrak{d}_{i}\left(\frac{1}{V}\right) \varphi(\tau) d \tau,
\end{gathered}
$$

where $i=1,2$ and $P, R \in \mathbb{R}^{n \times n}$ are symmetric matrices. If $\exists \gamma>0$ such that

$$
\begin{gathered}
G_{\mathfrak{d}_{i}}^{\top} P+P G_{\mathfrak{d}_{i}}>\gamma P, \quad P>0, \\
G_{\mathfrak{d}_{i}}^{\top} R+R G_{\mathfrak{d}_{i}}-\mu R>\gamma R, \quad R>0,
\end{gathered}
$$

then $\frac{\partial Q_{i}}{\partial V}<0$ for $\varphi \in C_{h}$ such that $\varphi \neq \mathbf{0} \in C_{h}$. The proof immediately follows from the formula

$$
\begin{gathered}
\frac{\partial Q_{i}}{\partial V}=-\frac{1}{V} \varphi^{\top}(0) \mathfrak{d}_{i}\left(V^{-1}\right)\left(H_{r_{i}} P+P H_{r_{i}}\right) \mathfrak{d}_{i}\left(V^{-1}\right) \varphi(0)+ \\
+\frac{(-1)^{i} \mu \int_{-h}^{0}\left(e V^{(-1)^{i}}\right)^{\frac{\mu(2 \tau+h)}{h}} \frac{2 \tau+h}{h} \varphi^{\top}(\tau) \mathfrak{d}\left(\frac{1}{V}\right) R \mathfrak{d}\left(\frac{1}{V}\right) \varphi(\tau) d \tau}{V}- \\
-\frac{\int_{-h}^{0}\left(e V^{(-1)^{i}}\right)^{\frac{\mu(2 \tau+h)}{h}} \varphi^{\top}(\tau) \mathfrak{d}\left(\frac{1}{V}\right)\left(H_{r} R+R H_{r}\right) \mathfrak{d}\left(\frac{1}{V}\right) \varphi(\tau) d \tau d \theta}{\leq-\gamma \frac{Q_{i}(V, \varphi)+1}{V}}
\end{gathered}
$$

Therefore, the functionals (8) are ILKF candidates and they satisfy Conditions $C 4^{*}$ ) and $C 6^{*}$ ) of Corollary 1 .

\section{Hyper Exponential CONTROL DESIGN FOR LINEAR SYSTEM}

\section{A. System Decomposition}

It is well-known (see, for example, [17]) that under Assumption 1 there exists a matrix $\Phi$ allowing the decomposition

$$
\Phi A \Phi^{-1}=\left(\begin{array}{ccccc}
\mathbf{0} & A_{12} & \mathbf{0} & \ldots & \mathbf{0} \\
\mathbf{0} & \mathbf{0} & A_{23} & \ldots & \mathbf{0} \\
\ldots & \ldots & \ldots & \ldots & \ldots \\
\mathbf{0} & \mathbf{0} & \mathbf{0} & \ldots & A_{(k-1) k} \\
A_{k 1} & A_{k 2} & A_{n 3} & \ldots & A_{k k}
\end{array}\right), \quad B_{0}=\Phi^{-1} B=\left(\begin{array}{c}
\mathbf{0} \\
\mathbf{0} \\
\ldots \\
\mathbf{0} \\
\tilde{B}
\end{array}\right) .
$$

where $k$ is the number of blocks (in both rows and columns) and $A_{i j} \in \mathbb{R}^{n_{i} \times n_{j}}$ are blocks of the transformed matrix $A$, such that $n_{1}+n_{2}+. .+n_{k}=n, n_{i-1} \leq n_{i}, \operatorname{rank}\left(A_{i+1}\right)=$ $n_{i}, \tilde{B} \in \mathbb{R}^{m \times m}, n_{k}=m$. By analogy with single-input control systems the corresponding block form can be called canonical. The simple recursive algorithm for construction of the matrix $\Phi$ can be found in [17].
Assumption 2: Let us assume that $\Phi$ transforms $L$ to a lower triangular block form, i.e.

$$
\Phi L \Phi^{-1}=\left(\begin{array}{ccccc}
L_{11} & \mathbf{0} & \mathbf{0} & \ldots & \mathbf{0} \\
L_{21} & L_{22} & \mathbf{0} & \ldots & \mathbf{0} \\
\ldots & \ldots & \ldots & \ldots & \ldots \\
L_{k 1} & L_{k 2} & L_{n 3} & \ldots & L_{k k}
\end{array}\right),
$$

where the blocks $L_{i j} \in \mathbb{R}^{n_{i} \times n_{j}}$ have the same dimensions as the corresponding blocks in the matrix $\Phi A \Phi^{-1}$.

\section{B. Main Result}

For $i=1,2$ let us introduce the linear dilations (see, [17])

$$
\mathfrak{d}_{i}(\lambda)=e^{G_{\mathfrak{o}_{i}} \ln \lambda} \quad \text { with } \quad \lambda>0,
$$

where

$$
G_{\mathfrak{d}_{i}}=\operatorname{diag}\left\{r_{i j} I_{n_{j}}\right\}
$$

$r_{i j}=1+(-1)^{i+1}(k-j) \mu, \mu \in\left(0, k^{-1}\right)$ and $I_{n_{j}} \in \mathbb{R}^{n_{j} \times n_{j}}$

- the identity matrices for $j=1,2, \ldots, k$. In [17] the dilations (10) were utilized for finite/fixed-time control design of delay-free control systems.

Let us define the control law as follows

$$
u\left(x_{h}(t)\right)=\tilde{B}^{-1} u_{0}\left(\Phi x_{h}(t)\right)+\tilde{u}\left(V(t), x_{h}(t)\right),
$$

where

$u_{0}(\varphi)=-\left(A_{k 1} A_{k 2} \ldots A_{k k}\right) \varphi(0)-\left(\begin{array}{lll}L_{k 1} & L_{k 2} \ldots L_{k k}\end{array}\right) \varphi(-h)$ and

with

$$
\tilde{u}(V, \varphi)=V^{1+(-1)^{i} \mu} K \mathfrak{d}_{i}\left(\frac{1}{V}\right) \Phi \varphi(0), \quad \varphi \in C_{h},
$$

$$
i=\left\{\begin{array}{ccc}
1 & \text { if } & Q_{1}\left(1, y_{h}(t)\right) \leq 1 \\
2 & \text { otherwise }
\end{array}\right.
$$

and $V$ is a solution to $Q_{i}(V, \varphi)=0$, where the functionals $Q_{i}$ are defined by (8), (9), (10).

It is worth stressing that due to conditions C4) and $\mathrm{C6}^{*}$ ) we have $i=1$ for $V \leq 1$ and $i=2$ for $V>1$.

If we denote $y=\Phi x$ then the cosed-loop system becomes

$$
\dot{y}(t)=\left(A_{0}+B K V^{1+(-1)^{i} \mu} \mathfrak{d}_{i}\left(\frac{1}{V}\right)\right) y(t)-L_{0} y(t-h),
$$

with

$$
Q_{i}\left(V, y_{h}(t)\right)=0, \quad i=\left\{\begin{array}{ccc}
1 & \text { if } & Q_{1}\left(1, y_{h}(t)\right) \leq 1 \\
2 & \text { otherwise }
\end{array}\right.
$$

where $A_{0}$ and $L_{0}$ are the block matrices $\Phi^{-1} A \Phi$ and $\Phi^{-1} L \Phi$ with zero blocks in the last row (due to $u_{0}$ ).

Let us calculate the time derivatives of the ILKF (8) along the trajectories of the latter system

$$
\frac{\partial Q_{i}\left(V, y_{h}(t)\right)}{\partial t}=2 y^{\top}(t) \mathfrak{d}_{i}\left(\frac{1}{V}\right) P \mathfrak{d}_{i}\left(\frac{1}{V}\right) \dot{y}(t)+\frac{d \mathcal{I}_{i}(t)}{d t},
$$

where $y_{h}(t) \in C_{h}: y_{h}(t)(\theta)=y(t+\theta), \theta \in[-h, 0]$ and

$$
\mathcal{I}_{i}(t)=\int_{t-h}^{t}\left(e V^{(-1)^{i}}\right)^{\frac{\mu(2(\tau-t)+h)}{h}} y^{\top}(\tau) \mathfrak{d}_{i}\left(\frac{1}{V}\right) R \mathfrak{d}_{i}\left(\frac{1}{V}\right) y(\tau) d \tau
$$

Let us denote $z(t)=\mathfrak{d}_{i}\left(\frac{1}{V}\right) y(t), s(t)=\mathfrak{d}_{i}\left(\frac{1}{V}\right) y(t-$ $h), L_{i}(V)=\mathfrak{d}_{i}\left(\frac{1}{V}\right) L_{0} \mathfrak{d}_{i}(V)$. Taking into account $\mathfrak{d}_{i}\left(\frac{1}{V}\right) A_{0} \mathfrak{d}_{i}(V)=V^{(-1)^{i} \mu} A_{0}$ we derive

$$
\begin{gathered}
2 y(t)^{\top} \mathfrak{d}_{i}\left(\frac{1}{V}\right) P \mathfrak{d}_{i}\left(\frac{1}{V}\right) \dot{y}(t)= \\
\left(\begin{array}{c}
z(t) \\
s(t)
\end{array}\right)^{\top}\left(\begin{array}{cc}
V^{(-1)^{i} \mu}\left(P(A+B K)+(A+B K)^{\top} P\right)-P L_{i}(V) \\
-L_{i}^{\top}(V) P & \mathbf{0}
\end{array}\right)\left(\begin{array}{c}
z(t) \\
s(t)
\end{array}\right)
\end{gathered}
$$


On the other hand, one has

$$
\frac{d \mathcal{I}_{i}(t)}{d t}=\frac{z^{\top}(t) R z(t)}{\left(e V^{(-1)^{i}}\right)^{-\mu}}-\frac{2 \mu \ln \left(e V^{(-1)^{i}}\right) \mathcal{I}_{i}(t)}{h}-\frac{s^{\top}(t) R s(t)}{\left(e V^{(-1)^{i}}\right)^{\mu}} .
$$

Since $\frac{d(1+\ln (\rho))}{d \rho} \leq \frac{d\left(\mu^{-1} \rho^{\mu}\right)}{d \rho}, \forall \rho \geq 1$ then $\ln \left(e V^{(-1)^{i}}\right) \leq \frac{V^{\mu(-1)^{i}}}{\mu}$ $\frac{\partial Q_{i}\left(V, y_{h}(t)\right)}{\partial t} \leq\left(\begin{array}{c}z(t) \\ s(t)\end{array}\right)^{\top} \Theta_{i}\left(\begin{array}{c}z(t) \\ s(t)\end{array}\right)-\frac{2 \mu \ln \left(e V^{(-1)^{i}}\right)\left(Q_{i}\left(V, y_{h}(t)\right)+1\right)}{h}$ where

$$
\Theta_{i}=\left(\begin{array}{cc}
V^{(-1)^{i} \mu}\left(P(A+B K)+(A+B K)^{\top} P+e^{\mu} R+\frac{2}{h} P\right) & P L_{i}(V) \\
L_{i}^{\top}(V) P & \frac{-R}{\left(e V^{(-1)^{i}}\right)^{\mu}}
\end{array}\right) .
$$

Therefore, if $\Theta_{i}<0$ and $P, R$ satisfy (9) then all conditions of Corollary 1 hold for $\alpha_{0}=\frac{\mu}{\gamma h}$ since $\frac{\partial Q_{i}}{\partial V} \leq-\gamma \frac{Q_{i}\left(V, y_{h}\right)+1}{V}$ due to Lemma 1. To guarantee $\Theta_{i}<0$ it is sufficient to ask

$$
\left(\begin{array}{cc}
P(A+B K)+(A+B K)^{\top} P+e^{\mu} R+\frac{2}{h} P & P L_{0} \\
L_{0}^{\top} P & \frac{-R}{e^{\mu}}
\end{array}\right) \leq 0
$$

together with $L_{i}(V) R^{-1} L_{i}^{\top}(V) \leq L_{0} R^{-1} L_{0}^{\top}$ for $V \leq 1$ if $i=1$ and for $V \geq 1$ if $i=2$.

Under Assumption 2 we can represent $L_{i}$ as follows:

$$
L_{i}(V)=\sum_{j=1}^{k-1} V^{(-1)^{i+1}} \mu(j-1) \Pi_{j} L_{0}
$$

where $\Pi_{j}$ is the projector to the block diagonal matrix with non-zero blocks only in $j$-th lower diagonal, namely,

$$
\begin{aligned}
& \tilde{L}_{1}:=\Pi_{1} L_{0}=\left(\begin{array}{ccccccc}
L_{11} & \mathbf{0} & \mathbf{0} & \ldots & \mathbf{0} & \mathbf{0} & \mathbf{0} \\
\mathbf{0} & L_{22} & \mathbf{0} & \ldots & \mathbf{0} & \mathbf{0} & \mathbf{0} \\
\mathbf{0} & \mathbf{0} & L_{33} & \ldots & \mathbf{0} & \mathbf{0} & \mathbf{0} \\
\ldots & \ldots & \ldots & \ldots & \ldots & \ldots & \ldots \\
\mathbf{0} & \mathbf{0} & \mathbf{0} & \ldots & L_{k-2} & \ldots & \\
\mathbf{0} & \mathbf{0} & \mathbf{0} & \ldots & \mathbf{0} & L_{k-1} & \mathbf{0} \\
\mathbf{0} & \mathbf{0} & \mathbf{0} & \ldots & \mathbf{0} & \mathbf{0} & \mathbf{0}
\end{array}\right),
\end{aligned}
$$

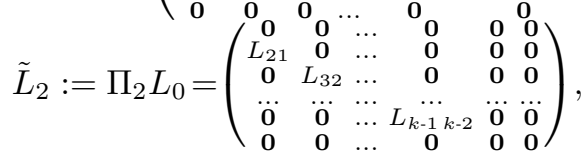

$$
\begin{aligned}
& \tilde{L}_{k-1}:=\Pi_{k-1} L_{0}=\left(\begin{array}{ccccc}
\mathbf{0} & \mathbf{0} & \mathbf{0} & \ldots & \mathbf{0} \\
\mathbf{0} & \mathbf{0} & \mathbf{0} & \ldots & \mathbf{0} \\
\ldots & \ldots & \ldots & \ldots & \ldots \\
L_{k-11} & \mathbf{0} & \mathbf{0} & \ldots & \mathbf{0} \\
\mathbf{0} & \mathbf{0} & \mathbf{0} & \ldots & \mathbf{0}
\end{array}\right) .
\end{aligned}
$$

Let us consider the positive definite matrix valued-function

$$
\Xi(\lambda)=\sum_{j=1}^{k-1} \lambda^{(j-1)} \tilde{L}_{j} R^{-1} \sum_{p=1}^{k-1} \lambda^{(p-1)} \tilde{L}_{p}^{\top},
$$

where $\lambda \in[0,1]$. Obviously, for $i=1,2$ one has

$$
L_{i}(V) R^{-1} L_{i}^{\top}(V)=\Xi\left(V^{(-1)^{i+1} \mu}\right) \text { and } L_{0} R^{-1} L_{0}^{\top}=\Xi(1) \text {, }
$$

where $V^{(-1)^{i+1} \mu} \in(0,1)$. Therefore, we have proven the next result.

Theorem 2: Let Assumptions 1-2 hold. If the system of matrix inequalities (9), (12) is feasible for some $P, R \in$ $\mathbb{R}^{n \times n}, K \in \mathbb{R}^{m \times n}$ and

$$
\Xi(1) \geq \Xi(\lambda) \quad \text { for all } \quad \lambda \in[0,1]
$$

then the origin of the closed-loop system (1), (11) is globally hyper exponentially stable with degree $r=1$ and convergence rate $\alpha=\left(\frac{\mu}{\gamma h}, 1\right)$.

The parametric inequality (15) can be checked numerically using a sufficiently dense grid on the segment [0,1]. A more conservative (but much more constructive) sufficient condition can also be provided. Indeed, to guarantee $\Xi(1) \geq$
$\Xi(\lambda)$ for $\lambda \in[0,1]$ it is sufficient to show $\frac{d}{d \lambda} \Xi(\lambda) \geq 0$ if $\lambda>0$. For this purpose let us rewrite $\Xi$ as follows

$$
\begin{gathered}
\Xi(\lambda)=\sum_{q=1}^{k-1} \lambda^{(2 q-2)} \tilde{L}_{q} R^{-1} \tilde{L}_{q}^{\top}+ \\
\sum_{j=1}^{k-1} \sum_{p>j} \lambda^{j+p-2}\left(\tilde{L}_{j} R^{-1} \tilde{L}_{p}^{\top}+\tilde{L}_{p} R^{-1} \tilde{L}_{j}^{\top}\right) .
\end{gathered}
$$

The latter identity immediately yields the next proposition.

Proposition 1: If for $1 \leq j<p \leq k-1$ the following system of matrix inequalities

$$
\begin{array}{ll}
0 \leq \tilde{L}_{j} R^{-1} \tilde{L}_{p}^{\top}+\tilde{L}_{p} R^{-1} \tilde{L}_{j}^{\top} & \text { if } j+p \text { is odd } \\
0 \leq \tilde{L}_{\frac{j+p}{2}} R^{-1} \tilde{L}_{\frac{j+p}{2}}^{\top}+\tilde{L}_{j} R^{-1} \tilde{L}_{p}^{\top}+\tilde{L}_{p} R^{-1} \tilde{L}_{j}^{\top} & \text { if } j+p \text { is even }
\end{array}
$$

holds then (15) is fulfilled.

Remark 1: If we denote $Z=R^{-1}, \beta>0, X=P^{-1}$ and $Y=K P^{-1}$ then the system of matrix inequalities (9), (12), (16) can be rewriten as the system of LMIs:

$$
\begin{aligned}
& \left(\begin{array}{cc}
A X+B Y+X A^{\top}+Y^{\top} B^{\top}+e^{\mu} L_{0} Z L_{0}^{\top}+\frac{2}{h} X & X \\
X & \frac{-Z}{e^{\mu}}
\end{array}\right) \leq 0 \\
& H_{r_{i}} X+X H_{r_{i}}-\mu X>\gamma X, \quad X>0, \quad i=1,2 \\
& 0 \leq \tilde{L}_{j} Z \tilde{L}_{p}^{\top}+\tilde{L}_{p} Z \tilde{L}_{j}^{\top} \quad \text { if } j+p \text { is odd, } \\
& 0 \leq \tilde{L}_{\frac{j+p}{2}} Z \tilde{L}_{\frac{j+p}{2}}^{\top}+\tilde{L}_{j} Z \tilde{L}_{p}^{\top}+\tilde{L}_{p} Z \tilde{L}_{j}^{\top} \text { if } j+p \text { is even, } \\
& 1 \leq j<p \leq k-1 \text {, }
\end{aligned}
$$

which immidiately follows from Schur complement (e.g. [18])

Note that the last two LMIs disappear if $k=2$ or if all non-diagonal blocks in $L_{0}$ are zeros (i.e. $L_{q p}=\mathbf{0}$ for $q \neq p$ ).

\section{Aspects of Practical IMPLEMENTATION}

\section{A. Sampled-Time ILF Control}

In order to realize the control algorithm (11) in practice we need to know Lyapunov-Krasovskii functional $V$, but it is defined implicitly. The ILKF control can be implemented for linear control systems that admit the on-line variation of the feedback gains. Indeed, for any fixed $V_{0}$ the control $\tilde{u}\left(V_{0}, s\right)$ defined by (11) becomes a static linear feedback. Let us denote

$$
\Pi(V):= \begin{cases}\left\{\varphi \in C_{h}: Q_{1}(V, \varphi) \leq 1\right\} & \text { if } \quad V<1 \\ \left\{\varphi \in C_{h}: Q_{2}(V, \varphi) \leq 1\right\} & \text { if } \quad V \geq 1\end{cases}
$$

Lemma 2: Let the conditions of Theorem 2 hold and

$$
u(t)=B_{0}^{-1} u_{0}\left(y_{h}(t)\right)+\tilde{u}\left(V_{0}, y_{h}(t)\right),
$$

where $\tilde{u}$ is defined by (11) with an arbitrary fixed positive number $V_{0} \in \mathbb{R}_{+}$. Then the ellipsoid $\Pi\left(V_{0}\right) \subset C_{h}$ is positively invariant set of the closed-loop system (1), (2), (19) and the origin is globally exponentially stable.

Proof. Let us consider the functional $V: C_{h} \rightarrow \mathbb{R}_{+}$ defined as $V=Q_{i}\left(V_{0}, \varphi\right)+1$, where $i=1$ if $V_{0}<1$ and $i=2$ if $V_{0} \geq 1$. Due to Proposition 1 it satisfy the condition $\sigma_{1}(\|\varphi(0)\|) \leq V(\varphi) \leq \sigma_{2}\left(\|\varphi\|_{h}\right)$ for some $\mathcal{K}_{\infty}$ functions $\sigma_{1}$ and $\sigma_{2}$. Since $\dot{V}\left(y_{h}(t)\right)=\frac{\partial Q\left(V_{0}, y_{h}(t)\right)}{\partial t}$ then repeating the proof of Theorem 2 we derive $\dot{V}\left(y_{h}(t)\right) \leq$ $-2 h^{-1} \ln \left(e V_{0}^{(-1)^{i}}\right) V\left(y_{h}(t)\right)$ along the trajectories of the closed-loop linear system (1), (2), (19).

In the next corollary we study the case where the value $V$ in (11) can be changed only in some sampled time instances 
$t_{i}>0$. In this case, the control law (11) becomes linear switched feedback.

Corollary 2: If 1) the conditions of Theorem 2 hold, 2) $\left\{t_{j}\right\}_{i=0}^{+\infty}$ is an arbitrary sequence of time instances:

$$
0=t_{0}<t_{1}<t_{2}<\ldots \quad \text { and } \quad \lim _{j \rightarrow+\infty} t_{j}=+\infty
$$

3) the control $u$ is defined as $u(t)=B_{0}^{-1} u_{0}\left(y_{h}(t)\right)+$ $\tilde{u}\left(V_{j}, y_{h}(t)\right)$ on each time interval $\left[t_{j}, t_{j+1}\right)$, where $\tilde{u}\left(V, y_{h}(t)\right)$ is defined by (11) and $V_{j} \in \mathbb{R}_{+}$: $Q_{i}\left(V_{j}, y_{h}\left(t_{j}\right)\right)=0$ with $i=1$ if $Q_{1}\left(1, y_{h}\left(t_{j}\right)\right)<1$ and $i=2$ otherwise, then the closed-loop system (1) is globally asymptotically stable.

Proof. Let $V$ be a Lyapunov-Krasovksii functional implicitly defined by the equation $Q_{i}(V, \varphi)=0$, where $i=1$ if $Q_{1}(1, \varphi)<0$ and $i=2$ otherwise. Note that $Q_{1}(V(\varphi), \varphi)=$ 0 if $V(\varphi) \leq 1$ and $Q_{2}(V(\varphi), \varphi)=0$ if $V(\varphi) \geq 1$ (see Corollary 1). Let us denote $V_{j}=V\left(y_{h}\left(t_{j}\right)\right)$.

I. To prove that the sequence $\left\{V_{j}\right\}_{j=1}^{+\infty}$ is monotone decreasing let us consider the time interval $\left[t_{j}, t_{j+1}\right)$ and the functional $\tilde{V}_{j}: C_{h} \rightarrow \mathbb{R}_{+}$defined $\tilde{V}_{j}(\varphi):=Q_{i}\left(V_{j}, \varphi\right)+1$ (with $i=1$ if $V_{j}<1$ and $i=2$ if $V_{j} \geq 1$ ), which (according to Lemma 2) is Lyapunov-Krasovski functional for the system (1), (2) with $u(t)=B_{0}^{-1} u_{0}\left(y_{h}(t)\right)+\tilde{u}\left(V_{j}, y_{h}(t)\right)$.

Repeating the proof of Lemma 2 we derive $\frac{d}{d t} \tilde{V}_{j}\left(y_{h}(t)\right) \leq$ $-2 h^{-1} \ln \left(e V_{j}^{(-1)^{i}}\right) \tilde{V}_{j}\left(y_{h}(t)\right)$ for $t \in\left[t_{j}, t_{j+1}\right)$. Hence, $\tilde{V}_{j}\left(y_{h}(t)\right)<\tilde{V}_{j}\left(y_{h}\left(t_{j}\right)\right)$ for all $\left(t_{j}, t_{j+1}\right]$ and

$$
\begin{gathered}
Q_{i}\left(V_{j}, y_{h}(t)\right)=\tilde{V}_{j}\left(y_{h}(t)\right)-1<\tilde{V}_{i}\left(y_{h}\left(t_{j}\right)\right)-1= \\
Q_{i}\left(V_{j}, y_{h}\left(t_{j}\right)\right)=0=Q_{i}\left(V\left(y_{h}(t)\right), y_{h}(t)\right), \\
t \in\left(t_{j}, t_{j+1}\right], \quad i=1,2 .
\end{gathered}
$$

For any fixed $\varphi \in C_{h} \backslash\{\mathbf{0}\}$ the functional $Q_{i}(\cdot, \varphi): \mathbb{R}_{+} \rightarrow \mathbb{R}$ is monotone decreasing (see Proposition 1). Then the latter chain of inequalities implies $V\left(y_{h}(t)\right)<V\left(y_{h}\left(t_{j}\right)\right)$ if $t \in$ $\left(t_{j}, t_{j+1}\right]$, i.e. the sequence $\left\{V_{j}\right\}_{j=1}^{+\infty}$ is monotone decreasing and $y_{h}(t) \in \Pi\left(V_{j}\right)$ for $t \geq t_{j}$. Moreover, $V\left(y_{h}(t)\right) \leq$ $V\left(y_{h}(0)\right)$ for all $t \geq 0$, i.e. the origin of the system (1) is Lyapunov stable.

II. Since the functional $V$ is positive definite then the monotone decreasing sequence $\left\{V_{j}\right\}_{j=1}^{\infty}$ (with $V_{j}=$ $V\left(y_{h}\left(t_{j}\right)\right)$ ) converge to some limit. Let us show now that this limit is zero. Suppose the contrary, i.e. $\lim _{j \rightarrow \infty} V_{j}=V_{*}>0$ or

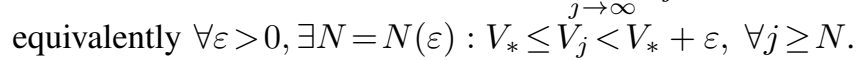

The function $\tilde{u}: \mathbb{R}_{+} \times C_{h} \rightarrow \mathbb{R}^{m}$ is continuous on $(0,+\infty) \times C_{h} \backslash\{\mathbf{0}\}$. Then we have

$$
\begin{gathered}
\left\|\tilde{u}\left(V_{j}, \varphi\right)-\tilde{u}\left(V_{*}, \varphi\right)\right\|= \\
\left\|V_{j}^{1+(-1)^{i} \mu} k \mathfrak{d}_{i}\left(V_{j}^{-1}\right) \varphi(0)-\left(V_{*}\right)^{1+(-1)^{i} \mu} k \mathfrak{d}_{i}\left(V_{*}^{-1}\right) \varphi(0)\right\| \\
\leq \sigma(\varepsilon)\|\varphi(0)\|, \quad \forall j \geq N,
\end{gathered}
$$

where $\sigma(\cdot) \in \mathcal{K}$ and $i=1$ if $V^{*}<1$ and $i=2$ if $V \geq 1$. This means that for $t>t_{N}$ the closed-loop system (1) can be presented in the form

$$
\dot{y}(t)=\left(A_{0}+B\left(K_{*}+\Delta(t, \varepsilon)\right)\right) y(t)-L_{0} y(t-h),
$$

where $K_{*}=V_{*}^{1-\mu} K D_{r}\left(V_{*}^{-1}\right)$ and $\Delta(t, \varepsilon) \in \mathbb{R}^{m \times n}$ : $\|\Delta(t, \varepsilon) y(t)\| \leq \sigma(\varepsilon)\|y(t)\|$.
Since according to Lemma (2) $u(t)=K_{*} y(t)$ is linear static stabilizing feedback then for sufficiently small $\varepsilon \in \mathbb{R}_{+}$ we have $y_{h}(t) \rightarrow 0$ as $t \rightarrow \infty$, i.e. there exists $t^{*} \geq t_{N}$ such that $\tilde{V}_{*}\left(t_{*}\right)=V_{*}$ and $\tilde{V}_{*}(t)<V_{*}$ for $t>t_{*}$. This contradicts with our assumption and means $\lim _{i \rightarrow \infty} V_{i}=0$ implying that the closed-loop system (1) with sampled-time implementation of the ILKF control algorithm is globally asymptotically stable.

The proven corollary guarantees that the ILFK control with sampled variation of $V$ guarantees asymptotic stabilization of the closed-loop system (1) independently on the sampling period.

\section{B. Digital Implementation}

The sampled ILKF control (11) implementation requires solving the equation $Q(V, \varphi)=0$ numerically and on-line in order to find an appropriate value of $V_{i}$ at the time instant $t_{i}$. Fortunately, for practical reasons rather simple numerical procedures can be utilized.

Denote as before $V_{j}:=V\left(t_{j}\right)$ and $y_{j}:=y_{h}\left(t_{j}\right)$ and suppose that the implicit part control is realized in the sampled way, i.e. $\tilde{u}\left(V_{j}, s\right)$ on the time interval $\left[t_{j}, t_{j+1}\right)$, where $0=t_{0}<t_{1}<t_{2}<\ldots$ and $\lim t_{j}=+\infty$.

\section{Algorithm 1:}

INITIALIZATION: $V_{0}=1 ; a=V_{\min } ; \quad b=1$;

STEP :

$$
\begin{aligned}
& \text { If } Q_{i}\left(1, y_{j}\right)+1>0 \text { then } i=2 \text { else } i=1 ; \\
& \text { endif; } \\
& \text { If } Q_{i}\left(a, y_{j}\right)+1>0 \text { then } a=b ; b=2 b ; \\
& \text { elseif } Q_{i}\left(a, y_{j}\right)+1<0 \text { then } b=a ; a=\max \left\{\frac{a}{2}, V_{\min }\right\} \\
& \quad \text { else } c=\frac{a+b}{2} ; \\
& \qquad \begin{array}{r}
\text { If } Q_{i}\left(c, y_{j}\right)+1 \text { then } b=c ; \\
\text { else } a=\max \left\{V_{\min }, c\right\} ;
\end{array}
\end{aligned}
$$

endif;

$V_{i}=b$;

If $y_{j} \in C_{h}$ is fixed and STEP of the presented algorithm is applied recurrently many times to the same $y_{j}$ then Algorithm 1 realizes:

1) a localization of the unique positive root of the equation $Q_{i}\left(V, y_{j}\right)=0$, i.e. $V_{j} \in[a, b]$, where $i=1$ if $Q_{1}\left(1, y_{j}\right)<1$ and $i=2$ otherwise;

2) improvement of the obtained localization by means of the bisection method, i.e. $(b-a) \rightarrow 0$.

Such an application of Algorithm 1 allows us to calculate $V_{j}$ with rather high precision but it requests a high computational capability of a control device. If the computational power is very restricted, then STEP of Algorithm 1 may be realized just once at each sampled instant of time. Lemma 2 proves that $\Pi_{\mu}\left(V_{j}, s\right)$ is an invariant set of the closedloop system (1), (11) with $u(t)=B_{0}^{-1} u_{0}\left(y_{h}(t)\right)+\tilde{u}\left(V_{j}, P\right)$. If the root of the equation $Q_{i}\left(V, y_{j}\right)=0$ is localized in $[a, b]$, Algorithm 1 always selects the upper estimate of $V_{i}$ providing that $y_{h}\left(t_{j}\right) \in \Pi\left(V_{j}\right)$, i.e. $V_{j}$ do not increase in time even when $y_{j}=y_{h}\left(t_{j}\right)$ varies in time.

The parameter $V_{\min }$ defines lower admissible value of $V$. In practice, this parameter cannot be selected arbitrary small 


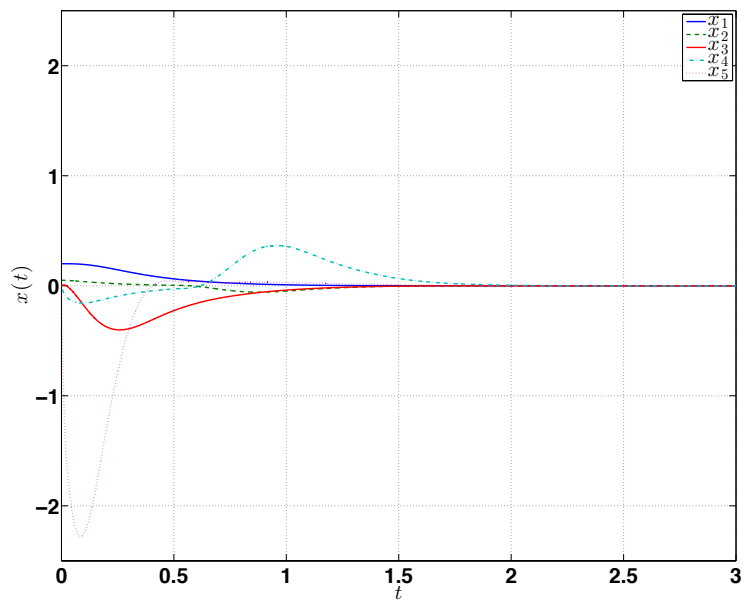

Fig. 2. Simulation results for ILFK control

due to finite numerical precision of digital devices.

\section{NumericAl EXAMPLE}

Let us consider the system (1) with $h=0.5$ and

$$
\begin{aligned}
& A=\left(\begin{array}{ccccc}
0 & 0 & 1 & 0 & 0 \\
0 & 0 & 0 & 1 & 0 \\
9 & 0 & -0.1 & 0 & 2 \\
0 & 19.6 & 0 & -0.3 & 0 \\
0 & 0.1 & 0 & 0 & -2
\end{array}\right), A_{d}=\left(\begin{array}{ccccc}
0 & 0 & 0 & 0 & 0 \\
0 & 0 & -1 & 0 & 0 \\
0.5 & 0 & 0.1 & 0 & 0 \\
0 & -0.3 & 0 & 1 & 0 \\
0 & 0 & 0 & 0 & 0.1
\end{array}\right), \\
& B=\left(\begin{array}{ll}
0 & 0 \\
0 & 0 \\
0 & 0 \\
1 & 0 \\
0 & 1
\end{array}\right), \quad \Phi=\left(\begin{array}{ccccc}
1 & 0 & 0 & 0 & 0 \\
0 & 0 & 1 & 0 & 0 \\
0 & -1 & 0 & 0 & 0 \\
0 & 0 & 0 & 1 & 0 \\
4.5 & 0 & 0 & -0.05 & 1
\end{array}\right),
\end{aligned}
$$

where the matrix $\Phi$ transforms the original system to the block forms with $n_{1}=1, n_{2}=n_{3}=2$ and

$$
\begin{gathered}
A_{12}=1, A_{23}=\left(\begin{array}{cc}
0 & 2 \\
-1 & 0
\end{array}\right), A_{31}=\left(\begin{array}{l}
0 \\
9
\end{array}\right), A_{32}=\left(\begin{array}{cc}
0 & 19.6 \\
4.4 & -0.1
\end{array}\right), \\
A_{33}=\left(\begin{array}{cc}
-0.3 & 0 \\
0 & -2.1
\end{array}\right), B_{0}=\left(\begin{array}{cc}
1 & 0 \\
0 & 1
\end{array}\right) \\
L_{11}=0, L_{21}=\left(\begin{array}{c}
0.5 \\
0
\end{array}\right), L_{22}=\left(\begin{array}{cc}
0.1 & 0 \\
1 & 0
\end{array}\right), \\
L_{31}=\left(\begin{array}{c}
0 \\
-0.475
\end{array}\right), L_{32}=\left(\begin{array}{cc}
0 & 0.3 \\
0 & 0
\end{array}\right), L_{33}=\left(\begin{array}{ll}
1 & 0 \\
0 & 1
\end{array}\right) .
\end{gathered}
$$

Using the system of LMIs (17) we derive

$$
\begin{aligned}
& P=\left(\begin{array}{ccccc}
38.7075 & 13.7738 & -0.2037 & 0.0300 & 2.3676 \\
13.7738 & 6.0479 & -0.1354 & 0.0201 & 1.1062 \\
-0.2037 & -0.1354 & 4.8892 & -0.8001 & -0.0330 \\
0.0300 & 0.0201 & -0.8090 & 0.2188 & 0.0049 \\
0.3676 & 1.062 & -0.0330 & 0.2049 & 0.0810
\end{array}\right) \\
& \left(\begin{array}{ccccc}
2.3676 & 1.1062 & -0.0330 & 0.0049 & 0.2810 \\
0.1331 & 0.0164 & 0.0000 & 0.0000 & 0.0011 \\
0.0164 & 0.6516 & -0.0006 & -0.0000
\end{array}\right) \\
& R=\left(\begin{array}{ccccc}
0.1331 & 0.0164 & 0.00000 & 0.00000 & 0.0011 \\
0.0164 & 0.6516 & -0.0006 & -0.0000 & 0.0433 \\
0.0000 & -0.0006 & 0.0770 & -0.0076 & -0.0002 \\
0.0001 & -0.000 & -0.006 & 0.077 & -0.000
\end{array}\right) \\
& \left(\begin{array}{ccccc}
0.0000 & -0.0006 & 0.0770 & -0.0076 & -0.0002 \\
0.0000 & -0.0000 & -0.0076 & 0.0477 & -0.0001 \\
0.0011 & 0.0433 & -0.0002 & -0.0001 & 0.0655
\end{array}\right)
\end{aligned}
$$

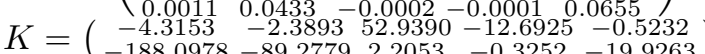

The Figure 2 depicts simulation results for the system (1) with the control (11) and the constant initial condition $\varphi(t)=$ $x_{0}$, where $x_{0}=(0.2,0.05,0,0,0)$. The numerical simulation has been done using the explicit Euler method with the sampling period $10^{-3}$. The implicit Lyapunov function has been calculated using Algorithm 1. The integral term in $Q_{i}$ has been approximated by the Simpson rule. As it was expected the simulation results show that realization of fast stabilization need large declinations (overshooting) of some variables during the transients (see, behavior of the variable $x_{5}$ on the Figure 2).

In order to compare the hyper exponential control with exponential (linear) one the numerical simulations have been
TABLE I

COMPARISON OF LINEAR AND ILKF CONTROLS

\begin{tabular}{|c|c|c|}
\hline & Linear Control & ILKF Control \\
\hline $10 x_{0}$ & $\|x(3)\|=0.0115$ & $\|x(3)\|=1.5134 \cdot 10^{-6}$ \\
$30 x_{0}$ & $\|x(3)\|=0.0344$ & $\|x(3)\|=1.6028 \cdot 10^{-6}$ \\
$100 x_{0}$ & $\|x(3)\|=0.1146$ & $\|x(3)\|=1.5787 \cdot 10^{-6}$ \\
$300 x_{0}$ & $\|x(3)\|=0.3439$ & $\|x(3)\|=1.5238 \cdot 10^{-6}$ \\
\hline
\end{tabular}

done also for linear stabilizing control $u=u_{0}+K x$, which is obtained from (11) for the fixed $V=1$. For linear control the simulations show that stabilization error essentially depended of the norm of initial condition, but in the ILKF case the stabilization error is uniformly (with respect to initial condition) bounded for $t \geq 3$. Such a behavior is similar to the fixed-time time stability that is well-known (see, e.g. [5], [19]) for delay-free systems.

\section{REFERENCES}

[1] A. M. Lyapunov, The general problem of the stability of motion. Taylor \& Francis, 1992.

[2] A. Polyakov, D. Efimov, W. Perruquetti, and J.-P. Richard, "Implicit Lyapunov-Krasovski Functionals for Stability Analysis and Control Design of Time-Delay Systems," IEEE Transactions on Automatic Control, vol. 60, no. 12, pp. 3344-3349, 2015.

[3] S. P. Bhat and D. S. Bernstein, "Geometric homogeneity with applications to finite-time stability," Mathematics of Control, Signals and Systems, vol. 17, pp. 101-127, 2005.

[4] W. Perruquetti, T. Floquet, and E. Moulay, "Finite-time observers: application to secure communication," IEEE Transactions on Automatic Control, vol. 53, no. 1, pp. 356-360, 2008.

[5] A. Polyakov, "Nonlinear feedback design for fixed-time stabilization of linear control systems," IEEE Transactions on Automatic Control, vol. 57(8), pp. 2106-2110, 2012.

[6] J. Moreno and M. Osorio, "Strict Lyapunov functions for the supertwisting algorithm," IEEE Transactions on Automatic Control, vol. 57, pp. 1035-1040, 2012.

[7] E. Cruz-Zavala, J. Moreno, and L. Fridman, "Uniform robust exact differentiator," IEEE Transactions on Automatic Control, vol. 56, no. 11, pp. 2727-2733, 2011.

[8] V. Andrieu, L. Praly, and A. Astolfi, "Homogeneous Approximation, Recursive Observer Design, and Output Feedback," SIAM Journal of Control and Optimization, vol. 47, no. 4, pp. 1814-1850, 2008.

[9] N. Nakamura, H. Nakamura, and Y. Yamashita, "Homogeneous stabilization for input-affine homogeneous systems," in Conference on Decision and Control, 2007, pp. 80-85.

[10] A. Levant, "Homogeneity approach to high-order sliding mode design," Automatica, vol. 41, no. 5, pp. 823-830, 2005.

[11] J.-P. Richard, "Time-delay systems: an overview of some recent advances and open problems," Automatica, vol. 39, no. 10, pp. 16671694, 2003.

[12] E. Fridman, Introduction to Time-Delay Systems: Analysis and Control. Springer, 2014.

[13] S. Choi and J. Hedrick, "Robust throttle control of automotive engines," ASME Journal of Dynamic Systems, Measurement, and Control, vol. 118, pp. 92-98, 1996.

[14] I. Karafyllis and Z.-P. Jiang, Stability and Stabilization of Nonlinear Systems. Springer, 2011.

[15] B. Driver, Analysis Tools with Applications. Springer, 2003.

[16] A. Polyakov, J.-M. Coron, and L. Rosier, "On finite-time stabilization of evolution equations: A homogeneous approach," in Conference on Decision and Control, 2016, pp. (https://hal.inria.fr/hal-01 371089 ).

[17] A. Polyakov, D. Efimov, and W. Perruquetti, "Robust stabilization of mimo systems in finite/fixed time," International Journal of Robust and Nonlinear Control, vol. 26, no. 1, pp. 69-90, 2016.

[18] A. Poznyak, Advanced Mathematical Tools for Automatic Control Engineers. Volume 1: Deterministic Technique. Elsevier, 2008.

[19] A. Polyakov, D. Efimov, and W. Perruquetti, "Finite-time and Fixedtime Stabilization: Implicit Lyapunov Function Approach," Automatica, vol. 51, no. 1, pp. 332-340, 2015. 